\title{
THE PARASTERNAL DEFECT
}

\author{
BY \\ E. F. CHIN AND E. R. DUCHESNE \\ From the Thoracic Surgical Unit, Southampton Chest Hospital
}

(RECEIVED FOR PUBLICATION APRIL 29, 1955)

The parasternal defect, or Morgagni hernia, may not be as rare as previous authors have stated. Brown (1952) gives its incidence as $3 \%$ of diaphragmatic defects, and Harrington (1951) in a review of 334 cases of diaphragmatic hernia found it in 14 only. In the mass radiography units at Portsmouth and Southampton 30 cases have been picked up and a further three cases have been found in patients who were admitted to the Surgical Unit for other conditions.

Since this defect causes symptoms in a small proportion of patients the majority do not come to medical scrutiny and investigation. The increasing use of mass radiography and our awareness of what this shadow probably is may lead to an increased recognition of Morgagni hernia. There is no doubt that many cases have been mis-labelled in the past, and they have often been designated as pleuro-pericardial cysts when indeed they were herniae of the foramen of Morgagni.

Most of the herniae through the diaphragm can be explained satisfactorily on an embryological basis, and in the majority of cases a certain error in embryological development will lead to the adult defect which would be expected both in situation and shape ; the size depends upon the severity of the developmental error.

We do not feel, however, that the herniae which occur anteriorly through the foramen of Morgagni have been satisfactorily correlated with regard to the anatomical findings at operation and the original embryological incoordination which probably caused them.

\section{EMBRYOLOGY}

The embryology of this deformity has been reviewed by several authors (Barrett and Wheaton, 1934 ; Brown, 1952).

Originally the embryonic disc is a flat ovoid, which develops as the roof of an ovoid cavity, the yolk sac. The embryonic plate becomes folded around the top of the yolk sac at the sides, anteriorly and posteriorly. Originally, the cardiogenic area of the embryonic plate lies at its most anterior joint and forms the apex of the area which folds downward anteriorly. This folding is carried on more at the front than at the sides and the rear, with the result that the cardiogenic area comes to lie ventrally. The diverticulum from the yolk sac, which lies above it, develops into the fore gut. This process is generally referred to as the "pericardial reversal."

At the same time as these events are taking place the intra-embryonic coelom is developing and the vascular system is being laid down, and by the time the pericardial reversal has been completed the vitelline and umbilical veins are present and entering the caudal end of the pericardium. This part of the pericardium consists then of primitive connective tissue, which with further development becomes the septum transversum.

By the time that the septum transversum has been formed, the lung buds have developed considerably and have invaded that part of the intraembryonic coelom which overlies them and becomes the pleural cavity. As the lungs increase in size they enlarge locally the primitive pleural cavities at the expense of the body wall, with the result that the pleural cavities become separated from the primitive peritoneal cavities by two folds of body wall situated laterally and caudally and known as the pleuroperitoneal folds.

The diverticulum from the yolk sac formed by pericardial reversal becomes the oesophagus. Dorsal to the primitive oesophagus there lies a considerable area of loose mesenchyme which becomes the meso-oesophagus.

Thus, to recapitulate, the early rudiments of the diaphragm are all present; ventrally the septum transversum, laterally the pleuroperitoneal folds, and dorsally the meso-oesophagus. These rudiments fuse together if all proceeds normally and form a connective tissue membrane which becomes the early diaphragm.

The septum transversum is a "busy area" and it takes part in the development of several organs : the anterior component of the diaphragm, the fibrous diaphragmatic aspect of the pericardium, 
and a large proportion of the liver together with the great veins entering these last two organs.

Caudally the liver is separated from the septum by encroachment of the coelom, which becomes the peritoneal cavity, while cephalad the pericardium and its contents become the middle mediastinum. The pericardial area on the superior surface of the diaphragm occupies a variable proportion of that surface, and its relative position may vary with reference to the anterior thoracic wall, which in full development is occupied by the sternum and its attachments, and this distance may be modified by the relative size and position of the liver at the time of stabilization.

Muscularization of the diaphragm probably begins at about the $3 \mathrm{~mm}$. stage and is synchronous with the actual definition of the various components. The muscles develop principally from the fourth cervical myotome, aided by the third and fifth. These myotomes, like the others forming the body wall, are divided into anterior and posterior components. The antero-lateral components form the muscles of the diaphragm and correspond to the lateral division of the hypomeres which form the transversus and intercostal layers. The dorsal portion of this muscle layer forms the crura and these correspond ontologically with the anterior spinal flexor group of muscles. The main point that we wish to emphasize here is that these myotomes invade the mesenchyme from dorsal to ventral and that the anterior part of the primitive diaphragm is the last part to receive its muscles.

The skeletal elements of the thorax are the ribs and sternum. The ribs are formed by the costal elements of the vertebrae which in the thoracic region grow out into the somatopleure or chest wall. The upper seven ribs eventually become joined together in sequence from before backwards by a bar of cartilage which fuses with its contralateral fellow and becomes the sternum at the ninth week of intra-uterine life. It is an established fact that the sternum fuses from before backwards, that is, above down, and that failures in fusion occur most commonly in the lower part and the resulting anomalies are bifid xiphoid, absent xiphoid, and sternal hiatus. It is not known for certain at what period the musculature of the diaphragm is complete, but it is reasonable to assume that it corresponds closely to the stage at which muscles derived from the same layer complete their fusion. The diaphragm corresponds ontologically with the transversus sheath which closes at about the seventh week of intrauterine life.
We suggest, therefore, that it is a reasonable precept that the coordination of the final muscularization of the diaphragm and the fusion of the xiphisternum may not be perfectly accurate and that the place where bony defect is known to occur is a reasonable situation to expect some degree of bony and muscular malalignment.

That herniae form through these defects and their formation is aided by the relatively rapid increase in the size of the abdominal contents before final diaphragmatic fusion is, we should like to submit, not a categorical statement of absolute facts, but an attempt to correlate two clinical findings with reference to the accepted embryological knowledge of the region concerned.

\section{SYMPTOMATOLOGY}

Thirty cases were seen as a result of mass radiographs and a further three were picked up in passing, but only six were operated on. Of the remaining 27 cases, only five had symptoms and these were in no way disabling. These patients complained of occasional discomfort in the right subcostal margin, occasional attacks of short, but sharp, pain below the right subcostal region on straightening up after bending down and usually associated with lifting something heavy. They

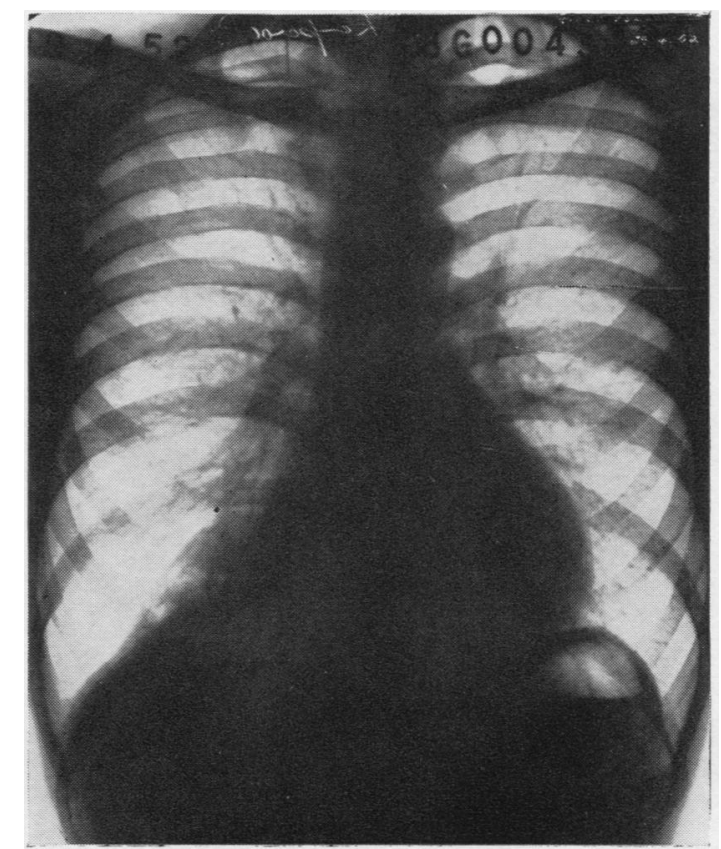

FIG. 1.-Radiograph of $\mathrm{a}^{-}$case with transverse colon in the sac; air in the sac is also seen. 


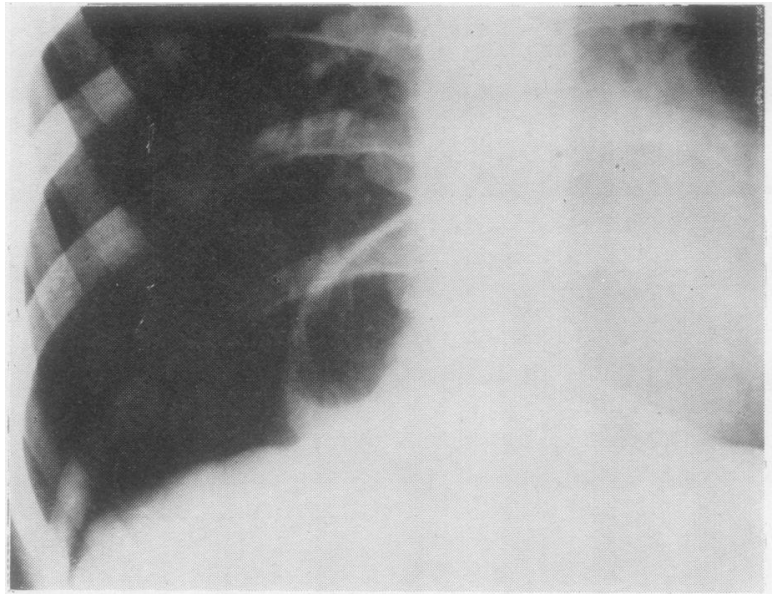

FIG. 2.-Demonstrates the fundus of the stomach in the sac and the air bubble is well seen.

also gave a history of vague sensations of discomfort below the right subcostal margin on lying down, but never severe enough to interfere with sleep. In none of these cases was there any justification to repair the hernia for symptoms.

The six patients operated on had symptoms. Two of these had the transverse colon in the sac (Fig. 1). They complained of intermittent attacks of colic, repeated bouts of abdominal distension, nausea, and sometimes vomiting. We would agree with Harrington (1951) that those cases with bowel in the sac have symptoms which are abdominal as opposed to respiratory. This is more common if the colon is in the sac. In both the patients with the colon in the sac the neck of the sac was small and the bowel was constricted as it passed through the defect. However, not all such cases have symptoms. One case was seen where the fundus of the stomach was a right-sided hernia, but this occasioned no symptoms and no surgery was undertaken (Fig. 2).

In two of the remaining four cases the sac contained omentum and in the other two cases there was no peritoneal sac, but extraperitoneal fat had slipped through the defect. The symptoms of these four patients followed a pattern which varied only in its intensity. They complained of right subcostal pain and discomfort. This pain was often associated with activity and seemed to be worsened when stooping and lifting. The pain could be severe, and although intermittent it was a daily occurrence. One case needs elaborating.

The patient was a woman in middle life, but was not particularly obese. For four years she had complained of a dragging pain in the right subcostal region. This was accentuated on exercise or when 7 she lay down. She suffered occasional attacks of $\frac{\bar{O}}{\partial}$ abdominal distension and belching which were not $\frac{\bar{\sigma}}{\omega}$. relieved in any way by prescribed medicines. During $\vec{\nabla}$ the last year the pain had spread from the right sub- $\varnothing$ costal margin to the back. It became pronounced when recumbent at night, but was relieved when walk- $\vec{\circ}$ ing around during the day. One day while stooping and picking up her shopping-basket she experienced $\vec{\omega}$ an intense pain below the right subcostal margin $\stackrel{\omega}{\sigma}$ which spread across the precordium and down the inner aspect of her left arm. She collapsed in the $\vec{x}$ street and was taken home by car. A diagnosis of $\vec{O}$ coronary thrombosis was made even though subse- it quent E.C.G.s, all leads being done, were normal. She was kept in bed for six weeks, and stated that $\vec{A}$ during this time the pain in the subcostal margin and 음 down the inner aspect of the arm were present all the time and became slightly more accentuated when $c^{2}$ lying flat at night. When allowed out of bed the $\mathbb{D}$ discomfort became less and she only experienced it $\mathbb{\mathbb { D }}$ when straightening up after stooping or when lying down in bed at night. A barium meal and chole- $\mathbb{\complement}$ cystograms were normal. A radiograph taken at that $\vec{\theta}$ time showed a typical foramen of Morgagni hernia of (Fig. 3). When admitted to this unit a pneumoperitoneum was performed and no air was admitted to the sac, as the omental contents were firmly adherent to all aspects of the sac. After the induction of the pneumoperitoneum the pain increased so much that morphia was necessary. The hernia was $\frac{\circ}{\varnothing}$ subsequently repaired, and she has been completely free from symptoms for three years.

It would appear that if the contents of the sac are extraperitoneal fat or omental fat within a? peritoneal sac, and that if either become adherent to the pericardial fat, symptoms may occur due to traction on either of these structures. The induction of a pneumoperitoneum in a case with adhe- $\frac{x}{0}$ sions in the sac causes severe pain which resembles 3 the type of pain with which the patient is afflicted on change of posture, but it is always much more intense. On three occasions it has required morphia to control the pain. Fig. 4 demonstrates a case before and after the induc- $\frac{D}{0}$ tion of a pneumoperitoneum and shows adhesions in the sac. This patient had intense discomfort o and pain following the pneumoperitoneum due to $N$ the dragging on the omentum.

It has also become apparent that in those $\omega$ patients where the omental contents drop out of the sac, and thus there are no adhesions, there is no pain after the pneumoperitoneum and no symptoms related to the diaphragmatic defect.

Thus, to recapitulate, transverse colon in the sac produces symptoms which are predominantly $\overrightarrow{\mathbb{D}}$ abdominal. If the defect is caused by either extra- $\frac{?}{\mathbb{D}}$ peritoneal fat or a peritoneal sac containing omen- $\frac{\varrho}{0}$ 


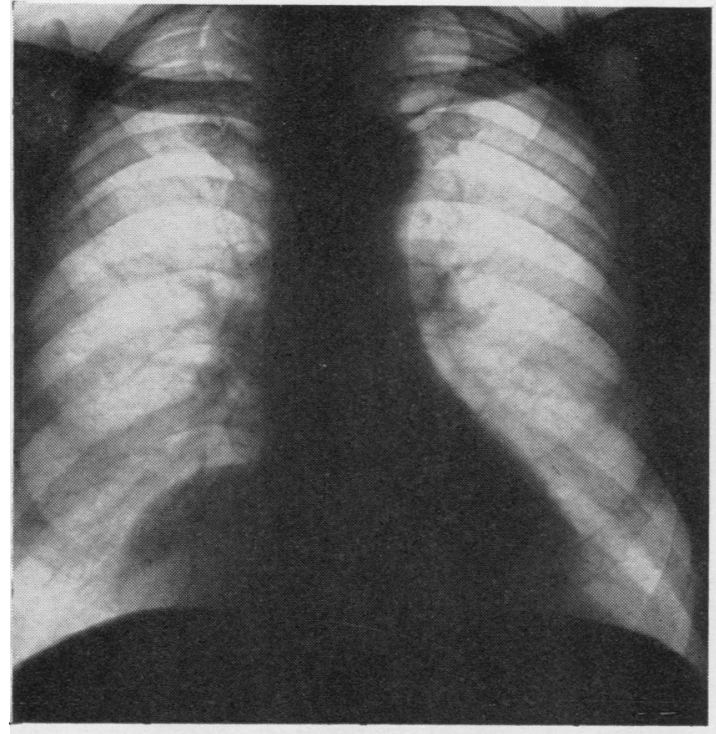

FIG. 3.-Radiographic findings in a typical hernia, demonstrating close contact to the anterior chest wall. This sac contained omentum and there is no air visible.

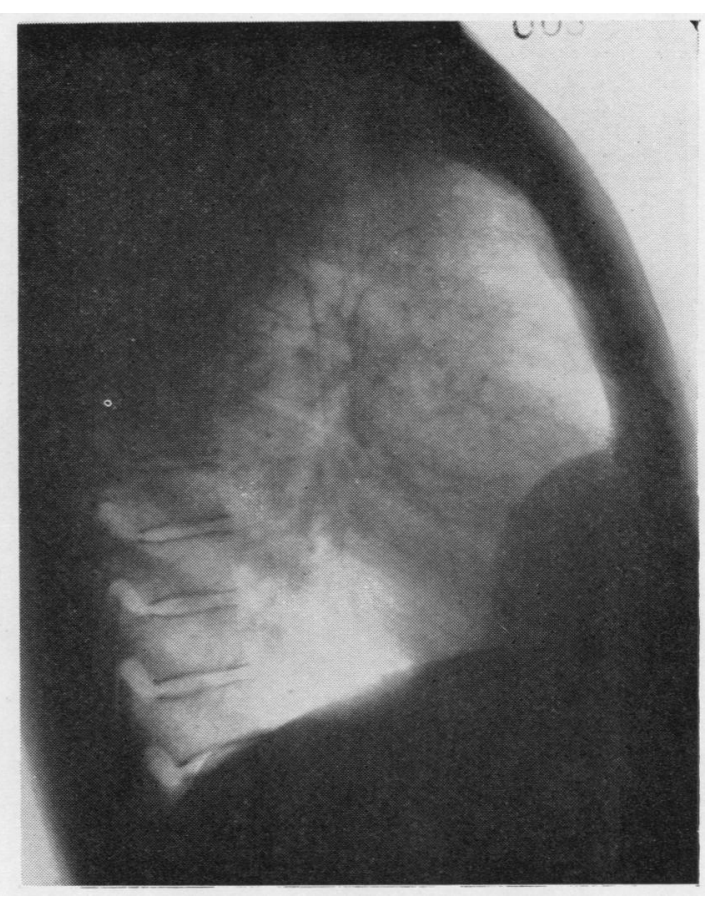

FIG. 4.-The omentum is partly adherent within the sac after the induction of a pneumoperitoneum.
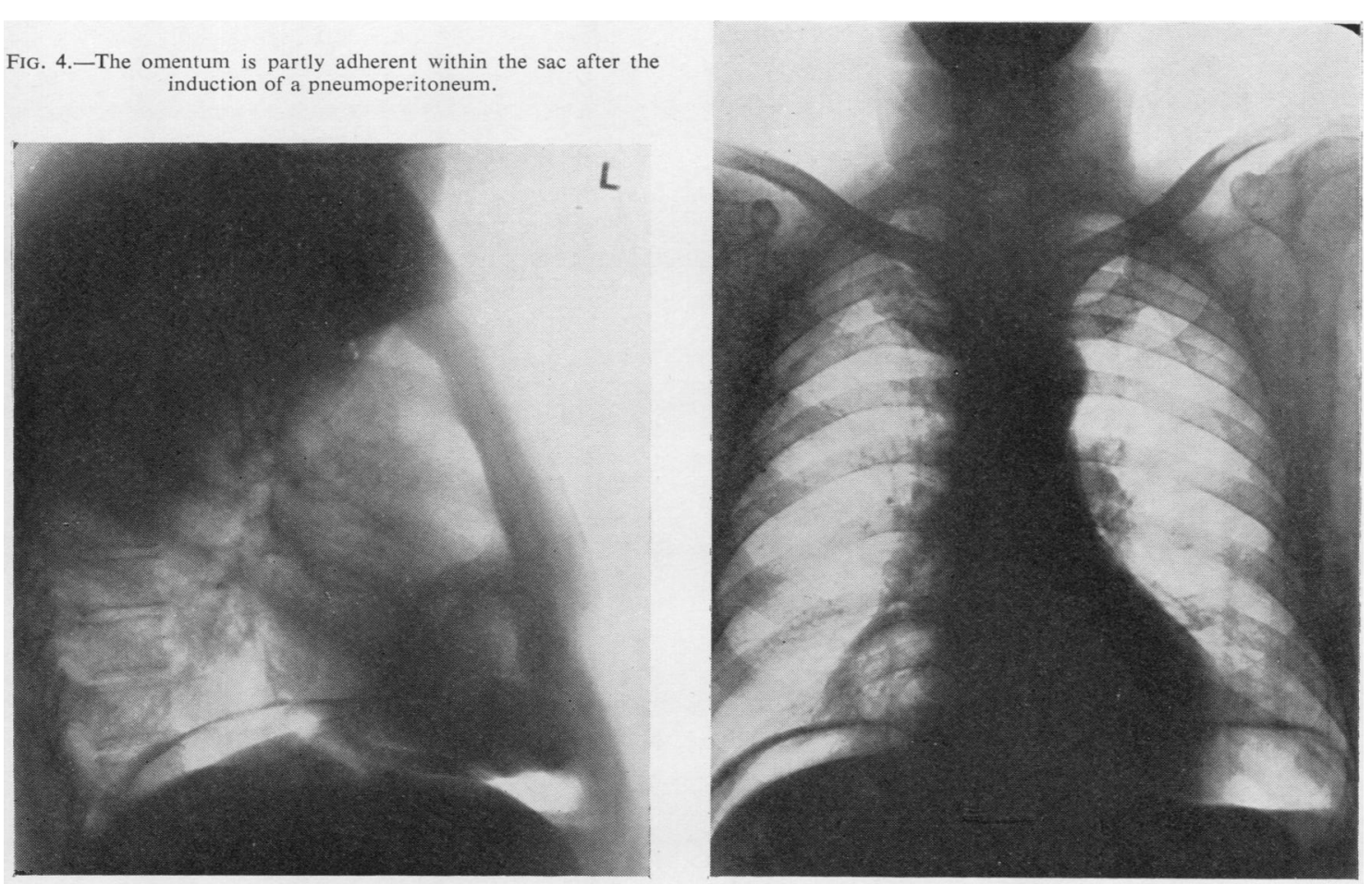


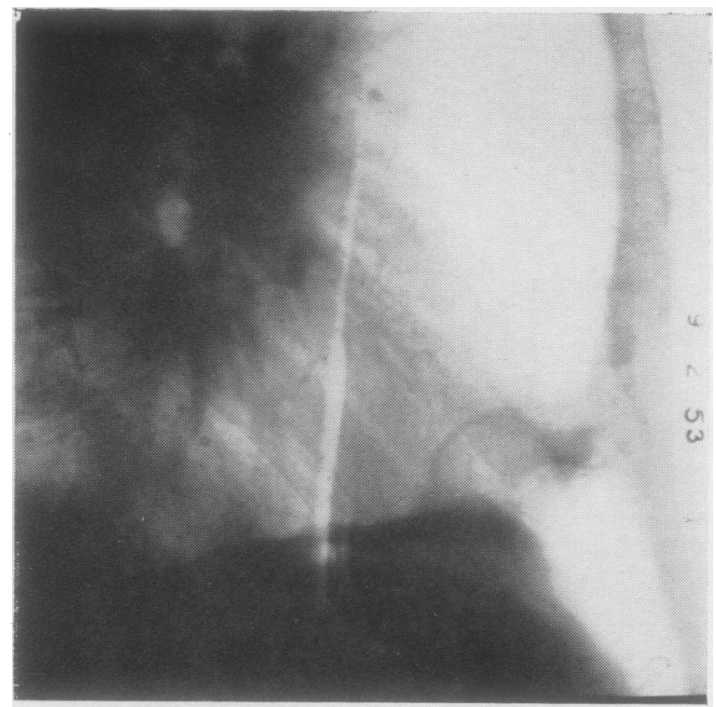

Fig. 5.-A small hernia which was symptomless is seen and pneumoperitoneum showed complete emptying of the sac.

tum and in either of these if the contents are adherent to the pericardial fat, then symptoms will occur. If there is no adherence then symptoms are unlikely. Fig. 5 demonstrates such a case.

\section{Diagnosis}

Diagnosis can often be made on a posteroanterior and lateral film. The radiograph is typical in that there is a rounded shadow in the cardiophrenic angle, usually on the right. In this series we have seen no case of a left-sided hernia demonstrated radiologically, though we have encountered one during the repair of an eventration. A lateral film is essential and this shows the shadow to be situated in the anterior costophrenic angle in close contact with the anterior thoracic wall (Fig. 4).

The P.A. view alone is not diagnostic, as this condition on a P.A. film can be simulated by a cold abscess of the spine, a localized eventration of the diaphragm, a mesothelial cyst of the diaphragm, a haemangioma of the mediastinum (Valle, 1954), or a pleuropericardial cyst.

If the contents going through the defect are extraperitoneal fat or omentum then the shadow in the chest is opaque, but if bowel is present then there is usually air in the mediastinal tumour (Figs. 1 and 2). In certain cases it is impossible to differentiate by plain films alone.

Proof can sometimes be obtained by a pneumoperitoneum. In this series a pneumoperitoneum was performed in 10 cases and a positive finding was obtained in six. In some patients the shadow does not fill with air because there is no peritoneal sac and the defect is occupied by extraperitonealo fat only, or the omental contents of the peritoneal sac are adherent to the neck and fundus of the $\frac{\text { s }}{\square}$ sac and the space completely obliterated.

A pneumoperitoneum is not without its dangers ; it can cause an acute exacerbation of the patient's $\overrightarrow{-}$ symptoms. In one case of pneumoperitoneum, the patient, standing upright, became very dis- $\vec{\omega}$ tressed and collapsed (fortunately just after the radiograph had been taken). He was admitted to the ward and appeared to have a cardiac tampo- $\overrightarrow{0}$ nade. The radiograph was quickly developed andic he was seen to have a pneumopericardium. The foot of the bed was elevated and air was taken off $\overrightarrow{+}$ with immediate relief. There is no doubt that this 은 man had the condition described by O'Brien (1939)as hernia diaphragmatica spuria centralis con- $\mathbb{D}$ genita. He also had a Morgagni hernia.

\section{ANatomy}

Normally the diaphragm rises from the lower $\vec{\bullet}$ surface of the last six ribs and costal cartilages, or the xiphoid process, and the first three lumbar vertebrae. The cases of anterior hernia that weo have seen are of two types. In the first there is a definite fibrotendinous slip arising from the xiphoid process, and the hernia passes between this $\frac{\mathrm{O}}{\mathrm{D}}$ slip on to the medial side and the muscular origin of the seventh costal cartilage which lies on its $\overrightarrow{\overrightarrow{0}}$ lateral side. The xiphoid process in these cases appears to be normal, and it is probable that this

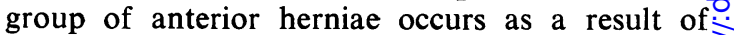
incomplete fusion of the pleuro-peritoneal folds and the ventral part of the septum transversum with consequent failure of muscularization of the latter component. In the second type the hernia 3 passes through a defect that starts on the left of the sternum and extends right across the midline $\frac{\mathrm{F}}{3}$ along the right seventh costal cartilage. In these cases the xiphoid is often small, asymmetric, and 5 in one case was absent; the anterior border of $D$ the pericardium is relatively more dorsal than usual, perhaps because of displacement by the N developing liver before the ninth week of intrauterine life. We suggest that this hernia is caused? by failure in the fusion of the xiphoid process $\omega$ with consequent failure of the anterior musculare elements to obtain an adequate area for attach $\frac{10}{?}$ ment.

In those cases operated on, four had the defect ${ }^{-\infty}$ extending across the midline to the left seventh costal cartilage so that virtually the anterior aspect of the defect was a small portion of the seventh costal cartilage on the left, the xiphoid and the $\mathbb{D}$ inner aspect of the right seventh costal cartilage, 
bounded behind by the rolled edge of the diaphragm and supported medially by the pericardium. In these cases the xiphoid was abnormal and rudimentary and in one case it was bifid.

By definition, a hernia must always have a sac, but it is not true to state that the contents within this defect of the diaphragm are always contained within a peritoneal sac. In three of our cases extraperitoneal fat had herniated through the defect and there was no peritoneal sac. Brown (1953) describes a case with extraperitoneal fatty contents. One case was found in association with an eventration of the diaphragm (Fig. 6). This was present on the left side and the contents were extraperitoneal fat. Thus, our findings differ from those of Meyer (1950) and Cruickshank, Goel, and Macedo (1954). who state that Morgagni herniae always have a sac.

\section{Surgical Repair}

The majority of cases do not require surgery, as they have no symptoms. It is felt, however, that any case with colon in the sac should be operated on whether symptoms are present or not, because the dangers of obstruction are real, as the neck of the sac is small and tight. In the two herniae containing colon that we have operated on there was a constriction around the bowel as it passed through the diaphragm. If the contents are fatty only, and repetitive and disabling discomfort is being produced, then a repair should be performed.

Of the six cases operated on, all have been relieved of their symptoms.

There is a divergence of opinion as to which is the best approach. We have used the abdominal approach in three cases and thoracotomy in three. In future all cases will be operated upon through a thoracotomy, as in our hands this is much the easier way of reducing the hernia and repairing the defect. The reasons for this are (1) the approach to the sac is direct and if there are adhesions to the pericardial fat they can be liberated under direct vision; (2) the defect in the diaphragm is readily repaired and both the subcostal margin and the rolled edge of the diaphragm are accessible; (3) if the patient is obese the approach through the abdomen can be difficult, and if the contents are firmly adherent to the pericardial fat then the incision may have to be carried across the subcostal margin into an intercostal space. This can be painful post-operatively.

The technique of repair is simple. We use 25 thread on a heavy needle and pick up the rolled muscular edge of the diaphragm and take a deep

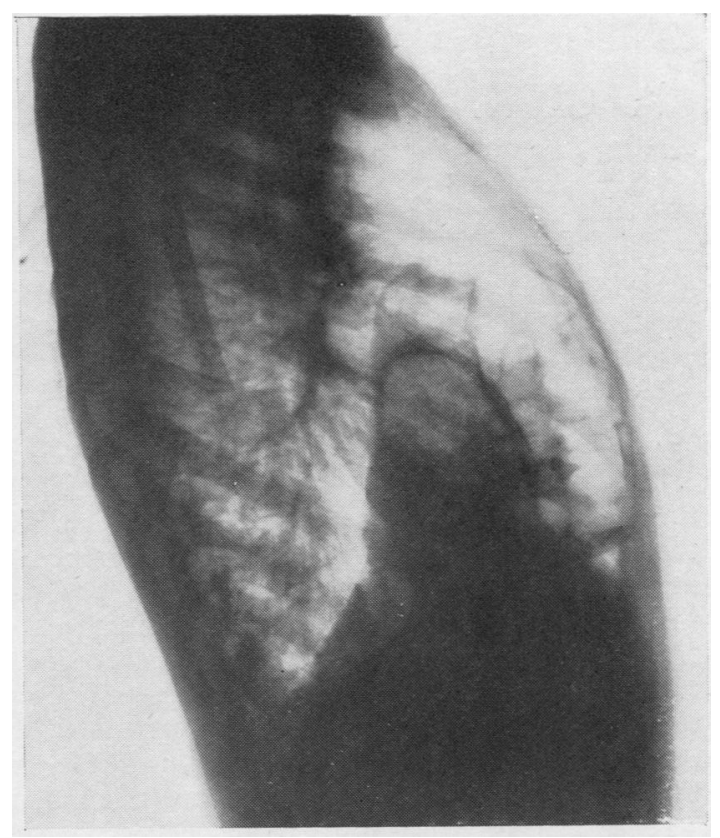

FIG. 6.-Case of eventration on the left with a small Morgagni hernia containing extraperitoneal fat not visible radiologically.

bite into the perichondrium. Four or five such sutures are required and the diaphragm and subcostal margin appose quite readily. Recurrence should be rare.

\section{SUMMARY}

Thirty-three cases of hernia through the foramen of Morgagni have been reviewed. Six cases were operated on for symptoms and a further three were found at thoracotomy for other conditions. It is felt that this type of diaphragmatic defect is more frequent than the literature suggests. Only a minority have symptoms. It may be necessary to carry out a repair for symptoms, and if bowel is in the sac this should always be done. Repair is best performed by the thoracic approach. The embryology of the defect is discussed.

I am very grateful to Dr. J. D. Lendrum and Dr. M. E. Moore, of the mass radiography units at Portsmouth and Southampton, for allowing me to review their cases, and I am particularly grateful to Dr. Lendrum for his help.

REFERENCES

Barrett, N. R., and Wheaton, C. E. W. (1934). Brit. J. Surg., 21,420 .

Brown, R. Warwick (1952). Thorax, 7, 266.

(1953). Ibid., 8, 162.

ruickshank, G., Goel, P. P., and Macedo, M. E. M. (1954). Brit. J. Tuberc., 48, 141 .

Harrington, S. W. (1951). Rev. Gastroent., 18, 243.

Meyer, H. W. (1950). J. thorac. Surg., 20, 235.

M'Brien, $\mathrm{H}$. (1939). J. Anat., Lond., 74, 131.

Valle, A. R. (1954). Ann. Surg., 140, 771 . 